Mitteilung aus Finsens med. Iysinstitut, Kopenhagen.

(Ilinik für Eautkrankheiten.)

\title{
Fehlende Wassermannsche Reaktion bei tertiärer Hautsyphilis.
}

Von

\author{
Chefarzt Axel Reyn.
}

Im allgemeinen besteht die Auffassung, dab das Blut eines Patienten mit tertiärer, nicht antiluetisch behandelter Hautsyphilis positive Wassermann-Reaktion gibt.

Diese Annahme ist wohl in der Regel richtig und in der von dem dänischen Arzte Boas ausgeführten großen und interessanten Arbeit über Wassermannsche Reaktion, wo die Patienten zum ersten Male in behandelte und nicht behandelte geteilt werden, berichtet er, daß bei 26 Patienten mit nichtbehandelter tertiärer Hautsyphilis kein einziges Mal negative Reaktion entstand.

Marcus, ${ }^{1}$ ) der nicht Hautsyphilis und Syphilis lokalisiert auf andere Stellen von einander getrennt hat, untersuchte 46 Patienten mit nicht-behandelter tertiärer Lues, und nicht bei einem einzigen fehlte die Reaktion.

Daß die Reaktion hin und wieder bei nicht-behandelter tertiärer Hautsyphilis ausbleiben kann, zeigen folgende 3 Krankengeschichten.

1. Mann, 52 Jahre. Früher stets gesund, verneint Lues. Im Januar 1910 begann das jetzige Leiden des Patienten mit Röte der Nase. Unter verschiedener äußerlicher Behandlung verschlimmerte sich das Leiden.

1) Archiv für Derm. a. Syphilis. Bd. CVII. 
Im Juli 1910 wandte er sich an Finsens med. Lysinstitat. Weiche Nase war geschwollen rot mit tiefen Narben, einzelnen kleinen Knötchen, Pustel und Wunden. Sonst keine Krankheitssymptome. WassermannReaktion negativ.

\section{Röntgen.}

25. August. Das Zentrum der Affektion hält sich zikatriziert, aber das Leiden hat sich auf den rechten Nasenflügel hinaus und etwas nach oben auf den Nasenrücken verbreitet, präsentiert sich wie kleine tiefe Wunden mit wallartiger Infiltration in der Peripherie. WassermannReaktion negativ.

\section{Versuchsweise Lichtbehandlung.}

6. Dezember 1910. Auf Nasenspitze und Nasenrücken diffuse Infiltration, kleine leicht versenkte Narben und Wunden. Linker Nasenflügel in seinem ganzen Umfange stark geschwollen mit etlichen kleinen Wunden, geringer Defekt des Randes.

Die Nasenhöhle: Auf rechter Seite der Scheidewand weit hinten eine Wunde.

Wassermann-Reaktion positiv, aber schwach $(0 \cdot 2 \mathrm{ccm} . .45)$ Jodkalium und Injekt. mit Salicyl. hydrag.

6 Wochen später war das Leiden vollkommen ausgeheilt.

2. Frau, 39 Jahre, Witwe. Früher stets gesund. Eine Geburt vor 13 Jahren. Das Kind lebt und ist gesund. Sie ist nur dieses eine Mal schwanger gewesen. Sie hat niemals Wunden oder Ausschlag um Genitalia oder auf der Haut beobachtet.

Vor $1 / 2$ Jahre bekam die Patientin einen Knoten in linker Wange vor den oberen Backenzähnen. Da man annahm, das Leiden stamme von den Zähnen, wurden 8 ausgezogen; jedoch wachs der Knoten und wurde deshalb geschnitten. Es wurde nur wenig Blut entleert.

Vor ca. 5 Monaten entstanden Wunden auf der Außenseite der Wange. Diese Wunden wurden größer und vor 2 Monaten machte man Wassermann-Reaktion, die negativ ausfiel.

Im März 1911 wandte sie sich an Finsens Lysinstitut. Sie war in der dazwischen liegenden Zeit mit Umschlägen und Salbe behandelt.

6. März 1911. Auf linker Wange eine kreisförmige Affektion von $5 \mathrm{~cm}$ Diameter (sieh beigefügtes Bild). Die Haut ist blaurot, infiltriert. Im Zentrum der Affektion beginnende Narbenbildung. Der Rand knotig mit einer Anzahl kleiner nierenförmigen Wunden. Die ganze Wange etwas geschwollen und in Sulcus alveolo-bucalis ein walnusgroßer Knoten. Bidigitale Untersuchung ergibt recht bedeutende Infiltrationen der ganzen Wange. In beiden Regionen inframaxiilare, einzelne kleine Drüsen, übrigens weder auf Haut- oder Schleimhaut kränkliche Veränderungen, ebenfalls ergibt 
die objektive Untersuchung im ganzen normale Verhältnisse. Urin ohne Eiweiß, ohne Zucker.

\section{Probeexzision.}

Blutprobe zn Wassermann.

Injekt. von $5 \mathrm{cg}$ Salicyl. hydrarg.

Die Wassermann-Reaktion war dieses Mal positiv $(0.1 \mathrm{ccm} 0)$

$(0.05 \mathrm{ccm} 30)$

Die Probeexzision ergab: Das Epithel etwas nekrotisch, zahlreiche Rundzellen, einzelne Riesenzellen und epitheloide Zellen.

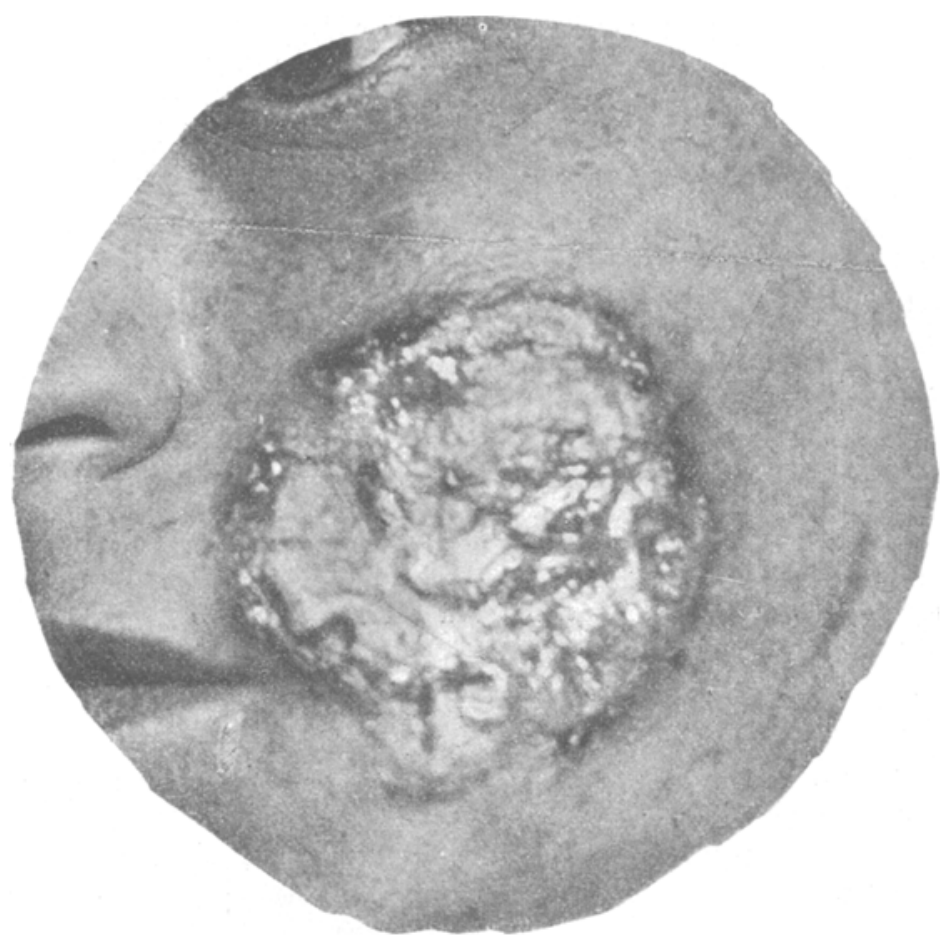

24. April 1910. Nach 13 Injektionen von $10 \mathrm{cg}$ salizylsaurem Quecksilber ist das Leiden vollständig ausgeheilt mit einer etwas einfallenden Narbe. Die Infiltration der Wange ist verschwunden.

3. 54jähr. Mann. Vor 24 Jahren Syphilis, mit 150 Schmierkuren behandelt. Die letzten 20 Jahre nicht behandelt. Im Nacken in der Haar- 
grenze und einige om aufwärts eine Anzahl knotige Infiltrationen, mit Schorfstückchen bedeckt, nach deren Entfernung zeigen sich kleine Wunden. Die Knötchen bestehen wesentlich aus Granulationsgewebe, die Wunden haben an einzelnen Stellen serpiginösen Charakter.

In beiden Handfächen ein tylotisches (schwielenartiges) Ekzem. Wassermann-Reaktion negativ.

Die Wunden wurden größer, und es bildeten sich, trotz verschiedener Behandlung, neue Knoten, weshalb 2 Monate später nochmals Wassermann gemacht wurde, der wiederum negativ ausfiel. Impfung auf Sporotrichose ergab ebenfalls negatives Resultat. 3 Monate, nachdem der Patient auf dem Institute in Behandlung genommen worden war, befand sich die Affektion in beständigem, aber langsamem Wachstum. Es waren nun 10 recht tiefe, gut 2pfenniggroße Wunden za sehen.

Der Patient bekam deshalb Jodkalium und Stichkuren. Nach $150 \mathrm{~g}$ Jodkalium und 10 Injektionen von $10 \mathrm{cg}$ salizylsaurem Quecksilber war das Leiden vollständig ausgeheilt.

Wir haben hier 3 Patienten mit nicht-behandelter tertiärer Hautsyphilis, wo Wassermann bei zweien erst bei wiederholter Untersuchung positive Reaktion ergab, während sie bei dem dritten dauernd negativ verblieb.

Dies zeigt, wie vorsichtig man sein soll, aus einer fehlenden W a ss er mann-Reaktion den Schluß zu ziehen, daß eine Affektion nicht Syphilis ist, wenn die übrigen Symptome dafür sprechen; und ich glaube, es muß gesagt werden, daß selbst, wenn die $W$ a s sermann sche Reaktion negativ ausfällt und sich bei wiederholten Untersuchungen negativ verhält, sich, wo das Aussehen und der klinische Verlauf eines Leidens tür Syphilis sprechen, eine antiluetische Kur empfiehlt, bevor zu anderer, mehr eingreifender Behandlung übergegangen wird.

In einer wie großen Prozentzahl aktiv nicht behandelter tertiärer Hautsyphilis die Reaktion fehlschlagen kann, weiß ich nicht, jedoch kommt es bei kleinen und wenig ausgebreiteten Affektionen nicht selten vor. In dem hiesigen Institute haben wir im ganzen 10 Fälle nicht-behandelter tertiärer Syphilis und bei 3 derselben versagte die Reaktion.

Diese Zahlen stimmen nicht mit den Resultaten überein, zu denen $\mathrm{Boas}$ in seimem Disputat gekommen ist. 
Boas, der, wie erwähnt, als erster seine Fälle in behandelte und nicht behandelte einteilt, hat im ganzen 26 Patienten mit nicht-behandelter Hautsyphilis, welche sämtlich positive Reaktion gaben.

Es läßt sich ja schwer sagen, was die eigentliche Ursache hierzu sein kann, aber sämtliche von Boa s') untersuchten Patienten hatten schwerere und mehr ausgebreitete Affektionen, sie hatten länger als die von mir besprochenen bestanden und vielleicht haben wir hierin die Begründung. Es ließe sich ja denken, daß eine tertiäre Syphilis einige Zeit aktiv gewesen sein muB, bevor im Blute so viele, der für die Reaktion notwendigen Stoffe gebildet werden, daß diese positiv wird; ana$\log \mathrm{dem}$, daß eine gewisse Zeit vergehen muß, bevor das Blut eines Patienten mit frischer Syphilis positive Reaktion gibt. Die 2 ersten Fälle sprechen ja stark dafür, weil sie beide, nachdem sie erst negative Reaktion ergaben, nach einigen Wochen positiv reagierten.

Der dritte Fall spricht nicht dagegen, denn es handelte sich ja um einen sehr wenig aktiven Prozeß, der trotz 3monatlicher Nicht-Behandlung keine erwähneuswerten Fortschritte machte.

Zum Schluß will ich bemerken, daß diese Fälle nicht das Vertrauen auf die Wassermann-Reaktion erschüttern dürfen, es ist ja gerade eine Seltenheit, daß sie versagt und wir haben in der Wassermann-Probe ein diagnostisches Hilfsmittel, welches unschätzbar ist und in zahlreichen Zweifel-Fällen die richtige Diagnose gibt, ja sicher viele Patienten vor unzweckmäBiger Behandlung bewahrt und sie nicht nur von ernsthaften Symptomen befreit, sondern sie gleichzeitig vor kïftigem Unglücke sichert.

1) Mündliche Mitteilung des Herrn Dr. med. B oas. 
Auf dem hiesigen Lichtinstitut gelang es uns einige Male, bei Patienten die lupusartige Symptome aufwiesen, durch eine positive Wassermann-Reaktion zu der richtigen Diagnose zu gelangen. ${ }^{x}$ )

1) Ove Strandberg: Über die Bedeutung der WassermannReaktion in der Rhinologie. Berlin. klin. Wochenschr. 1911. 